\title{
A randomized clinical trial evaluating the immunomodulatory effect of convalescent plasma on COVID-19-related cytokine storm
}

\author{
Mandana Pouladzadeh ${ }^{1} \cdot$ Mehdi Safdarian $^{2}(1) \cdot$ Peyman Eshghi $^{3,4} \cdot$ Hassan Abolghasemi ${ }^{5}$. Alireza Ghorbani bavani ${ }^{6}$. \\ Behnam Sheibani ${ }^{7}$. Parastoo Moradi Choghakabodi ${ }^{8}$ (1) Abdolaziz Feghhi ${ }^{9} \cdot$ Mehri Ghafourian Boroujerdnia $^{10}$. \\ Arash Forouzan ${ }^{11}$. Mohammad Ali Jalali Far ${ }^{8}$. Gholam Abbas Kaydani ${ }^{12}$. Elham Rajaei ${ }^{13} \cdot$ Mansour Amin $^{14,15}$. \\ Mehdi Torabizadeh ${ }^{16} \cdot$ Farid Yousefi ${ }^{15} \cdot$ Reza Hadaddezfuli ${ }^{17}$
}

Received: 11 December 2020 / Accepted: 30 March 2021 / Published online: 10 April 2021

(c) Società Italiana di Medicina Interna (SIMI) 2021

\begin{abstract}
Evaluating the effect of convalescent plasma (CP) on some cytokine storm indices in severe COVID-19 patients. Totally, 62 patients were randomly assigned into two groups for this clinical trial. Patients in the intervention group received one unit $(500 \mathrm{~mL})$ plasma on the admission day plus standard drugs while the controls merely received standard treatments. Eventually, primary and secondary outcomes were evaluated. In the CP group, compared with controls, the mean levels of lymphocytes and IL-10 significantly increased while the levels of IL-6, TNF- $\alpha$, and IFN- $\gamma$ decreased $(p<0.05)$. The length of in-hospital stay, and mortality rate did not significantly reduce in the CP group compared with controls $(p>0.05)$ while WHO severity scores remarkably improved $(p=0.01)$, despite the higher frequency of underlying diseases among the CP group (66.7\%) vs. controls (33.3\%). Although CP has a remarkable immunomodulatory and antiviral potential to improve the cytokine storm and disease severity in COVID-19 patients, it did not considerably affect the mortality rate.
\end{abstract}

Keywords Convalescent plasma $\cdot$ COVID-19 $\cdot$ Cytokine storm

\section{Introduction}

Coronavirus disease 2019 (COVID-19) was proclaimed as a critical global pandemic by the World Health Organization (WHO) in March 2020 caused by severe acute respiratory syndrome coronavirus 2 (SARS-Cov-2). The recent increasing evidence indicates higher levels of inflammatory cytokines in critical and severe COVID-19 patients than moderate and healthy groups. This "cytokine storm" can also indicate a poor prognosis, and may increase the mortality rate in COVID-19 patients. [1-4] The T cell over activation and an acute respiratory distress syndrome (ARDS) were discovered after the postmortem pathology examination of the lung that belonged to a dead COVID19 patient. [4] This cytokine storm can cause ARDS following the both alveolar epithelial and endothelial cell

Mehdi Safdarian

msafdaryan@gmail.com

Parastoo Moradi Choghakabodi parastoomoradi40@yahoo.com

Extended author information available on the last page of the article apoptosis, and vascular leakage that eventually may lead to death. [5] Pathological evidence suggests that the cytokine storm in COVID-19 infection are mainly caused by the immune response of antigen-presenting cells (APCs) and $\mathrm{T}$ cells against viral infection. [4] To date, various clinical studies have shown considerable cytokine disorders (proinflammatory and inflammatory cytokines, and chemokines) in COVID-19 patients. SARS-CoV-2 induced a high-level secretion of IL-6, IL-10, IL-2, and IL-4, tumor necrosis factor-alpha (TNF- $\alpha$ ), interferon-gamma (IFN- $\gamma$ ), C reactive protein (CRP), [2, 6] IL-8, MCP-1, IP-10, MIP-1a, MIP-1b, GM-CSF, G-CSF, and RANTES, [1] especially in severe patients. However, an opposite result has also been reported in Chen et al.'s study; they reported that SARS-CoV-2 infection reduced IFN- $\gamma$ expression by $\mathrm{CD} 4+\mathrm{T}$ cells in severe patients compared to moderate cases. [7] Of course, if a larger sample size $(n>21)$ was used, the results were more reliable.

To date, various potential treatments for modulating cytokine storm in COVID-19 have been evaluated in many clinical studies, including IL-6 Inhibition by tocilizumab (TCZ), [8-10] glucocorticoid therapy by corticosteroids, 
$[11,12]$ hydroxychloroquine (HCQ), [13, 14] and intravenous immunoglobulin (IVIG). In addition, the role of PD-1 checkpoint-inhibitor in reviving the lymphocytes energy and cytokine-adsorption devices (extracorporeal membrane oxygenation) to decrease inflammatory mediators in COVID19 patients is under discussion. [15] However, to date, no clinical trial has been reported on PD- 1 checkpoint-inhibitor and IVIG for COVID-19 treatment. Recently, Rieder et al. have reported a considerable reduction of IL- 6 after using CytoSorb $^{\circledR}$ adsorber for several COVID-19 patients. [16] Furthermore, several studies have shown that traditional medicines can relieve symptoms, shorten the length of hospital stay, and reduce the mortality rate in COVID-19 patients. [17, 18] For example, the extracts from artemisininfamily drugs could induce anti-inflammatory effects by modulating the immune system function. [19] Immunomodulatory therapy may alleviate hyper-inflammation symptoms in severe COVID-19 patients by affecting cytokines production. However, clinical experience with immunomodulatory agents in viral disease, their efficient dose, and side effects are still debatable. Therefore, in this regard, the potential treatments need more clinical evidence to validate.

Previously, the efficiency and safety of CP therapy have been confirmed as an emergency intervention in several pandemics, including the flu, West Nile virus, SARS-CoV, and Ebola virus. [20-23] To date, several clinical trials demonstrated that early transfusion of CP into severe COVID-19 patients after symptoms onset led to a reduction in mortality rate compared with other routine therapies. [24-30] Based on Piechotta et al.'s review, 14 clinical trials have reported some serious adverse events after convalescent plasma therapy. The majority of participants only reported one serious event limited to the first $4 \mathrm{~h}$ after CP transfusion. Fifteen deaths were reported, which probably were related to transfusion. Other serious adverse events were mainly allergic, e.g., anaphylaxis, dyspnoea, and acute lung injury. However, it is not obvious whether $\mathrm{CP}$ transfusion caused these serious adverse events or not. [31].

The convalescent plasma comprises a wide variety of blood-derived components, including neutralizing antibodies (NAbs), organic and inorganic compounds, water, and a great number of various proteins (coagulation factors, albumin, etc.). [32] In this respect, CP may induce immunomodulatory effects via the infusion of NAbs (IgG, IgM) and anti-inflammatory cytokines that blockade the inflammatory cytokines, complement, and autoantibodies in recipient patients.[33].

In the present clinical trial, we investigated the impact of convalescent plasma (CP) therapy on modulation of cytokine storm in severe COVID-19 patients. In this regard, we comparatively evaluated the changes in the levels of some inflammatory indices of cytokine storm in CP-treated patients and the treated group by routine drugs. The $\mathrm{CP}$ is collected by apheresis from survivors who previously infected by COVID-19 and developed antibodies against SARS-Cov- 2 .

\section{Methods}

\section{Trial design}

This hospital-based, parallel-group and randomized controlled trial was approved by the Ethics Committee of Ahvaz Jundishapur University of Medical Sciences, Ahvaz, Iran with Ethical Code: IR.AJUMS.REC.1399.003 and IRCT20200310046736N1. Eligible patients who referred to the Emergency department (ED) in Razi hospital of Ahvaz between March and May 2020 were enrolled for this study.

The inclusion criteria for $\mathrm{CP}$ donors:

1- Recovered individuals in the age range 20-45 years with a recovery asymptomatic period for at least 2 weeks.

2- Negative SARS-CoV-2 RT-qPCR test result.

3- The negative test result for Hepatitis B, C, AIDS, syphilis, HTLV-1, and influenza.

4- Donors with no IgA deficiency and or other dangerous underlying diseases.

5- No smokers.

6- No pregnant and lactating women.

7- Those who signed the informed consent.

8- All plasma donors prior showed the strong positive results of the SARS-CoV-2 IgG/IgM Quick Test (German) for neutralizing $\mathrm{IgG}$ antibodies and negative results for IgM antibodies.

The inclusion criteria for $\mathrm{CP}$ recipient were:

1- COVID-19 patients who had specified COVID-19 symptoms (less than 7 days since the onset of the symptoms).

2- The positive results of PCR test and CT scan.

3- Severity WHO score $>4$.

4- Blood oxygen saturation $\left(\mathrm{SPO}_{2}\right) \leq 93 \%$ in room air.

5- Individuals who no exhibit hypersensitivity to plasma intravenous administration.

6- Those who voluntarily signed the informed consent.

CP was obtained by apheresis according to National Standards for Blood Transfusion Service and blood bank guidelines.

\section{Interventions}

Every eligible patient referred to the hospital was randomly assigned via a table of randomized block designs at a 1:1 ratio to receive the standard treatment (Chloroquine 
phosphate, Lopinavir/Ritonavir, etc.) with or without intravenously administration of $500 \mathrm{ml} \mathrm{CP}$ on admission day. The first plasma unit was injected in the first $4 \mathrm{~h}$ after admission; according to the physician's recommendation, the second unit was prescribed if no improvement was observed after $24 \mathrm{~h}$.

Before beginning the clinical trial, nasopharyngeal swabs and serum samples were collected from all eligible patients for RT-qPCR and laboratory tests, respectively. In fact, their illness severity and improvement WHO score, and laboratory data were recorded in two steps: (1) on admission day and (2) at the day of discharge.

\section{Outcomes}

The primary outcome was the improvement in the levels of cytokine storm indices. Secondary outcomes were length of in-hospital stay (LOS), 2-month mortality after admission, the improvement in the 8-point WHO severity score, and the frequency of CP therapy-related side effects.

The "cytokine storm" is defined as a high concentration of pro-inflammatory cytokines in severely ill patients.

\section{Sample size}

Given that no similar trial done about the changes in serum cytokines pre- and post-CP therapy in COVID-19 patients so far, and according to the preliminary results of the pilot study of our colleagues (Abolghasemi et al., 2020), at least 30 cases for each group were allocated to achieve an average level. Cases in this study were patients with COVID-19 who met the inclusion criteria.

\section{Randomization, blinding and allocation}

This parallel-group, single-blind, and randomized controlled trial was designed by randomly assigning patients to the intervention and control groups using a 6-item randomized block method and an equal allocation ratio (1:1). To maintain single blinding, we used a "Simple Central randomization" in which the individual recruiting the patient (senior physician responsible for therapeutic intervention) contacted the center by phone after the patient is enrolled. The respondent in the center was the second researcher, who had designed a table of the 6-item randomized block by computer and added concealment codes without knowing the patients' medical conditions. However, the senior physician and patients were aware. In fact, the senior physician started the treatment process on the advice of the second researcher at the central randomization office.

\section{Statistical analysis}

SPSS software version 26 was used for statistical analysis. Kolmogorov-Smirnov test was used to evaluate the normality of variables. Categorical variables were compared using the Chi-square test and presented as frequency and percentages while continuous variables were compared with independent $t$ test and paired $t$ test, and presented as the mean \pm SD (standard deviation). Multivariate analysis of variance (MANOVA) was used to compare the simultaneous mean of the studied biomarkers in two groups. A $p$ value less than 0.05 is considered as statistically significant.

\section{Results}

Totally, 62 eligible COVID-19 patients were assigned to this clinical trial. All patients were in the secondary infection phase, i.e., pulmonary and hyperinflammatory stage presenting with the symptoms of persistent cough, shortness of breath, and low oxygen levels. Two patients were excluded from the study due to voluntary leave the follow-up step after receiving treatments and discharge (one from the intervention and another one from the control arm). Therefore, the 60 eligible patients were randomized and divided into two equal groups, i.e., eventually, 30 cases were followed-up and analyzed in each study group (Fig. 1).

\section{Baseline data}

Table 1 summarizes the demographic, clinical, and laboratory characteristics, as well as the primary and secondary outcomes for both studied groups. There was no significant difference between the intervention and control groups in terms of age and sex $(p=0.79)$. The mean age in the intervention group was $53.5 \pm 10.3$ years which was not significantly different from the mean age of the control group $57.2 \pm 17$ years. The frequency of underlying disease in the intervention group [20 (66.6\%)] was significantly more than the control group $[10(33.3 \%)](p$ value $=0.02 *)$. The most common underlying diseases were, respectively, diabetes mellitus, hypertension, and ischemic heart disease (IHD). In some cases, asthma, rheumatoid arthritis (RA), and hyperlipidemia were found. Three and five patients in the intervention and control groups needed to intubation. It is noteworthy that these intubated patients were eventually died. Five patients required the administration of the second unit of plasma. All patients received similar antiviral therapy, including Ritonavir/Lopinavir, and chloroquine phosphate. The types of patients' blood groups were often $\mathrm{A}[\mathrm{A}+(19)$, $\mathrm{A}-(3)], \mathrm{B}+(18),[\mathrm{O}+(17), \mathrm{O}-(1)]$, and $\mathrm{AB}+(2)$. 
Fig. 1 Patients flow diagram

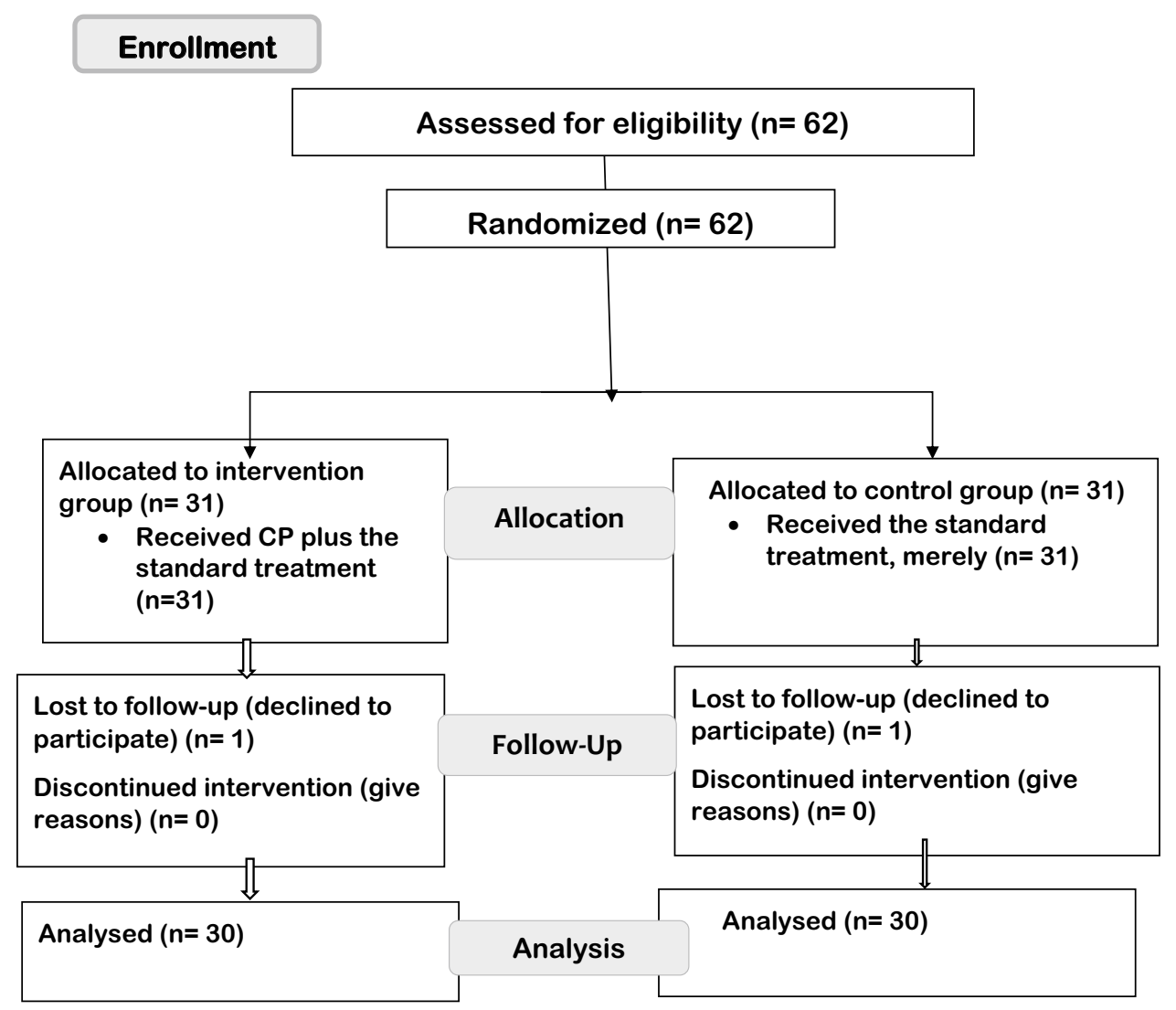

\section{Clinical and laboratory features of patients}

Clinical outcomes and changes in the levels of biomarkers, especially COVID-19-related cytokines (IL-6, TNF- $\alpha$, and IFN- $\gamma$ ), are presented in Table 2. No statistically significant differences in baseline levels of biomarkers were found between the two groups on the admission day $(p>0.05)$, except for IL- 6 and IFN- $\gamma$. The mean base levels of IL- 6 and IFN- $\gamma$ in the intervention group were significantly higher than their levels in controls $(p<0.05)$. The intragroup analysis indicated a significant increase in the level of absolute lymphocytes (per $\mathrm{mm}^{3}$ ) in the intervention group after CP therapy ( $p$ value $=0.001)$ while it did not significantly change in the control group ( $p$ value $=0.38$ ). In addition, $\mathrm{CP}$ therapy, compared to the routine treatments, significantly increased the mean level of absolute lymphocytes ( $p$ value $=0.012$ ).

Based on an intragroup analysis, a significant reduction was found in the levels of CRP, cytokines (IL-6, TNF- $\alpha$, and IFN- $\gamma$ ), and WHO severity score in the intervention group after CP therapy $(p<0.05)$; by contrast, their levels did not significantly change in the control group $(p>0.05)$, except for CRP. The level of ESR reduced insignificantly in the intervention group after CP therapy ( $p$ value $=0.13$ ), and a partially significant reduction in the mean (SD) difference of its level pre- and post-treatment was observed in the intervention group compared with controls ( $p$ value $=0.10$ ). Intragroup analysis indicated that the level of IL-10 significantly increased in CP-treated group $\left(p\right.$ value $\left.=0.015^{*}\right)$ while its level insignificantly increased in the standard treated group ( $p$ value $=0.35$ ). However, based on the outgroup analysis, its mean difference pre- and post-treatment was not significantly different between the two groups ( $p$ value $=0.15$ ). The length of in-hospital stay was not significantly different between both groups $(p$ value $=0.06$ ) However, the survival time during hospitalization for three deaths in CP-treated group was significantly longer than this time for five deaths in the control group ( $p$ value $=0.002$ ).

\section{Efficacy and safety}

Based on the MANOVA, there was a significant difference in the two groups for all biomarkers at the beginning of the study. Of course, by eliminating the effect of underlying diseases from the two groups $(p$ value $=0.042)($ Table 2$)$. Two factors, i.e., IL- 6 and IFN- $\gamma$, have become a significant factor between the two groups (Table 3 ). At the end of the study, the levels of biomarkers were compared again between the two groups by eliminating the effect of the underlying diseases. As a result, there was a significant difference between the two groups by considering all the factors $(p$ value $=0.004)($ Table 4$)$. The mean level of TNF- $\alpha$ 
Table 1 Comparison of demographic, clinical and laboratory characteristics, primary and secondary outcomes between case and control groups

\begin{tabular}{|c|c|c|c|}
\hline Demographic & $\begin{array}{l}\text { Case }(n=30) \\
n(\%)\end{array}$ & $\begin{array}{l}\text { Control }(n=30) \\
n(\%)\end{array}$ & $p$ value 1 \\
\hline \multicolumn{4}{|l|}{ Gender } \\
\hline Male & $16(53.3)$ & $17(56.7)$ & \multirow[t]{2}{*}{0.79} \\
\hline Female & $14(46.7)$ & $13(43.3)$ & \\
\hline \multicolumn{4}{|l|}{ Age (years) } \\
\hline$\leq 50$ & 11(36.7) & $12(40)$ & \multirow[t]{2}{*}{0.79} \\
\hline$>50$ & $19(63.3)$ & $18(60)$ & \\
\hline \multicolumn{4}{|l|}{ Underlying disease } \\
\hline Yes & $20(66.7)$ & $10(33.3)$ & \multirow[t]{2}{*}{0.01} \\
\hline No & $10(33.3)$ & $20(66.7)$ & \\
\hline \multicolumn{4}{|c|}{ Severity grade of COVID-19 } \\
\hline \multicolumn{4}{|l|}{ Pre } \\
\hline 5 & $20(66.7)$ & $25(83.3)$ & \multirow[t]{2}{*}{0.13} \\
\hline 6 & $10(33.3)$ & $5(16.7)$ & \\
\hline \multicolumn{4}{|l|}{ Post } \\
\hline$<5$ & 11(36.7) & $8(26.7)$ & \multirow[t]{3}{*}{0.34} \\
\hline 5 & $16(53.3)$ & $15(50)$ & \\
\hline$\geq 6$ & $3(10)$ & $7(23.3)$ & \\
\hline \multicolumn{4}{|l|}{ Died } \\
\hline Yes & $3(10)$ & $5(16.7)$ & \multirow[t]{2}{*}{0.44} \\
\hline No & $27(90)$ & $25(83.3)$ & \\
\hline Index evaluation & $\begin{array}{l}\text { Case group } \\
\text { mean } \pm \text { SD }\end{array}$ & $\begin{array}{l}\text { Control group } \\
\text { mean } \pm \text { SD }\end{array}$ & $p$ value 1 \\
\hline \multicolumn{4}{|l|}{ LOS } \\
\hline Total & $8.66 \pm 3.94$ & $6.66 \pm 4.30$ & 0.06 \\
\hline \multicolumn{4}{|l|}{ Died } \\
\hline No & $8.50 \pm 4.11$ & $7.32 \pm 4.31$ & 0.32 \\
\hline Yes & $10.3 \pm 0.57$ & $3.4 \pm 2.3$ & 0.002 \\
\hline \multicolumn{4}{|c|}{ Severity and improvement (WHO score) } \\
\hline Pre & $5.33 \pm 0.48$ & $5.16 \pm 0.38$ & 0.14 \\
\hline Post & $4.76 \pm 0.97$ & $4.93 \pm 1.01$ & 0.51 \\
\hline$p$ value 2 & $0.002 * *$ & 0.1287 & \\
\hline Difference & $-0.56 \pm 0.935$ & $-0.23 \pm 0.81$ & 0.14 \\
\hline Laboratory markers & $\begin{array}{l}\text { Case group } \\
\text { mean } \pm \text { SD }\end{array}$ & $\begin{array}{l}\text { Control group } \\
\text { mean } \pm \text { SD }\end{array}$ & $p$ value 1 \\
\hline \multicolumn{4}{|c|}{ Absolute lymphocyte (mm3) } \\
\hline Pre & $1545.6 \pm 786.96$ & $1631.53 \pm 913.43$ & 0.69 \\
\hline Post & $2282.11 \pm 1152.46$ & $1760.93 \pm 943.14$ & 0.06 \\
\hline$p$ value 2 & $0.0012 * *$ & 0.386 & \\
\hline Difference & $-675.55 \pm 969.33$ & $-106.79 \pm 654.15$ & $0.012 *$ \\
\hline \multicolumn{4}{|l|}{$\mathrm{CRP}(\mathrm{mg} / \mathrm{L})$} \\
\hline Pre & $59.75 \pm 28.015$ & $46.136 \pm 26.97$ & 0.06 \\
\hline Post & $27.38 \pm 20.25$ & $27.63 \pm 21.01$ & 0.96 \\
\hline$p$ value 2 & $<0.0001 * * *$ & $0.0008^{* * *}$ & \\
\hline Difference & $-31.6 \pm 30.1$ & $-17.95 \pm 25.7$ & 0.07 \\
\hline \multicolumn{4}{|l|}{$\operatorname{ESR}(\mathrm{mm} / \mathrm{hr})$} \\
\hline Pre & $68.1 \pm 31.30$ & $59.66 \pm 30.16$ & 0.29 \\
\hline Post & $55.27 \pm 31.10$ & $62.58 \pm 32.36$ & 0.40 \\
\hline$p$ value 2 & 0.1377 & 0.5607 & \\
\hline
\end{tabular}


Table 1 (continued)

\begin{tabular}{|c|c|c|c|}
\hline Laboratory markers & $\begin{array}{l}\text { Case group } \\
\text { mean } \pm S D\end{array}$ & $\begin{array}{l}\text { Control group } \\
\text { mean } \pm \text { SD }\end{array}$ & $p$ value 1 \\
\hline Difference & $-11.41 \pm 38$ & $-2.93 \pm 26.8$ & 0.10 \\
\hline \multicolumn{4}{|l|}{ IL-6 (pg/ml) } \\
\hline Pre & $51.23 \pm 11.259$ & $45.1 \pm 4.57$ & $0.007 * *$ \\
\hline Post & $42.25 \pm 6.55$ & $46.70 \pm 8.45$ & $0.02 *$ \\
\hline$p$ value2 & $<0.001$ & 0.1102 & \\
\hline Difference & $-8.98 \pm 10.35$ & $1.60 \pm 5.34$ & $<0.001 * * *$ \\
\hline \multicolumn{4}{|l|}{ IL-10 (pg/ml) } \\
\hline Pre & $17.33 \pm 7.34$ & $18.12 \pm 8.82$ & 0.71 \\
\hline Post & $21.82 \pm 12.06$ & $19.4 \pm 10.05$ & 0.40 \\
\hline$p$ value 2 & $0.015^{*}$ & 0.35 & \\
\hline Difference & $4.486 \pm 9.579$ & $1.28 \pm 7.43$ & 0.15 \\
\hline \multicolumn{4}{|l|}{ TNF- $\alpha(\mathrm{pg} / \mathrm{ml})$} \\
\hline Pre & $39.243 \pm 17.98$ & $39.03 \pm 16.30$ & 0.96 \\
\hline Post & $32.748 \pm 20.89$ & $41.79 \pm 22.27$ & 0.11 \\
\hline$p$ value 2 & $0.029^{*}$ & 0.4410 & \\
\hline Difference & $-6.49 \pm 15.56$ & $2.79 \pm 19.35$ & $0.045^{*}$ \\
\hline \multicolumn{4}{|l|}{ IFN- $\gamma(\mathrm{pg} / \mathrm{ml})$} \\
\hline Pre & $27.047 \pm 18.99$ & $24.79 \pm 11.28$ & $0.034^{*}$ \\
\hline Post & $18.55 \pm 9.92$ & $23.57 \pm 10.96$ & 0.61 \\
\hline$p$ value 2 & $0.0056^{* *}$ & 0.3265 & \\
\hline Difference & $-8.49 \pm 15.52$ & $-1.21 \pm 6.65$ & $0.0217 *$ \\
\hline
\end{tabular}

Mean (SD) difference between pre- and post-treatment

LOS length of in-hospital stay

$p$ value 1: comparing the mean levels of biomarkers between two groups based on an independent samples $t$ test

$p$ value2: comparing the mean levels of biomarkers in each group at the baseline and the day of discharge based on a paired $t$ test

Table 2 Comparison of mean vector components of biomarkers in case and control groups in starting study

\begin{tabular}{llllll}
\hline Multivariate tests $^{\mathrm{a}}$ & & & & & \\
\hline Effect & Value & $F$ & df & Error df & $p$ value \\
\hline Underlying diseases & & & & & \\
Pillai's trace & 0.180 & 1.594 & 7 & 51 & 0.158 \\
Wilks' lambda & 0.820 & 1.594 & 7 & 51 & 0.158 \\
Hotelling's trace & 0.219 & 1.594 & 7 & 51 & 0.158 \\
Roy's largest root & 0.219 & 1.594 & 7 & 51 & 0.158 \\
Group & & & & & \\
Pillai's trace & 0.239 & 2.287 & 7 & 51 & 0.042 \\
Wilks' lambda & 0.761 & 2.287 & 7 & 51 & 0.042 \\
Hotelling's trace & 0.314 & 2.287 & 7 & 51 & 0.042 \\
Roy's largest root & 0.314 & 2.287 & 7 & 51 & 0.042 \\
\hline
\end{tabular}

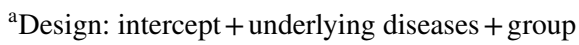

in the intervention group decreased from 39.24 to 27.62 while it increased slightly from 39 to 39.95 in the control group and a significant difference was observed between the two groups $(p$ value $=0.004)$ (Table 5). In addition, the mean level of IL-6 in the intervention group decreased from 51.23 to 42.58 while it increased slightly from 45.1 to 45.89 in the control group and a significant difference was observed between the two groups at the end of the study ( $p$ value $=0.014)($ Table 5$)$. However, its level was significantly different between the two groups at the beginning of the study. The difference in the mean level of IFN- $\gamma$ was also significant at the beginning and end of the study between the two groups.

Eventually, 3 (10\%) and $5(16.7 \%)$ patients died in the $\mathrm{CP}$ therapy group and control group, respectively. These deceased patients also required intubation before dying. The improvement in the 8-point WHO severity score was found among 16 (53.33\%) patients in the CP group and 8 (26.66\%) patients in the control group, respectively. Overall, CP therapy was not significantly associated with a shorter length of in-hospital stay (LOS) (HR 0.368 (0.020-6.838); $p=0.502$ ) and reduction in mortality rate (aOR $0.305,95 \%$ CI 0.009-10.065, $p=0.505$ ) while it remarkably improved the 8-point WHO severity score (aOR 7.314, 95\% CI $1.622-32.969, p=0.01$; Supplementary materials). CP therapy had not any serious side effects on patients. 
Table 3 Comparison of mean components of biomarkers in case and control groups with MANOVA analysis in starting study

\begin{tabular}{|c|c|c|c|c|c|c|c|}
\hline \multicolumn{8}{|c|}{ Tests of between-subject effects } \\
\hline Source & Dependent variable & \multirow{2}{*}{$\begin{array}{l}\text { Type III sum of } \\
\text { squares }\end{array}$} & \multirow{2}{*}{$\begin{array}{l}\mathrm{df} \\
1\end{array}$} & \multirow{2}{*}{$\begin{array}{l}\text { Mean square } \\
1014392.408\end{array}$} & \multirow{2}{*}{$\begin{array}{l}F \\
1.405\end{array}$} & \multicolumn{2}{|l|}{$p$ value } \\
\hline Underlying diseases & $\begin{array}{l}\text { Absolute } \\
\text { lymphocyte } \\
\text { per day }\end{array}$ & & & & & 0.241 & \\
\hline & $\begin{array}{l}\text { CRP (mg/L/ } \\
\text { day) }\end{array}$ & 26.414 & 1 & 26.414 & 0.034 & 0.854 & \\
\hline & $\begin{array}{l}\mathrm{ESR}(\mathrm{mm} / \mathrm{hr} / \\
\text { day) }\end{array}$ & 1599.430 & 1 & 1599.430 & 1.714 & 0.196 & \\
\hline & IL6 (pg/ml) & 253.752 & 1 & 253.752 & 3.591 & 0.063 & \\
\hline & $\begin{array}{l}\text { IL10 (pg/ml/ } \\
\text { day) }\end{array}$ & 26.696 & 1 & 26.696 & 0.401 & 0.529 & \\
\hline & $\mathrm{TNF} \alpha(\mathrm{pg} / \mathrm{ml})$ & 118.326 & 1 & 118.326 & 0.397 & 0.531 & \\
\hline & $\mathrm{IFN} \gamma(\mathrm{pg} / \mathrm{ml})$ & 316.875 & 1 & 316.875 & 2.998 & 0.089 & \\
\hline \multirow[t]{7}{*}{ Group } & $\begin{array}{l}\text { Absolute } \\
\text { lymphocyte } \\
\text { per day }\end{array}$ & 421860.208 & 1 & 421860.208 & 0.584 & & 0.448 \\
\hline & $\begin{array}{l}\text { CRP (mg/L/ } \\
\text { day) }\end{array}$ & 2644.224 & 1 & 2644.224 & 3.438 & & 0.069 \\
\hline & $\begin{array}{l}\mathrm{ESR}(\mathrm{mm} / \mathrm{hr} / \\
\text { day) }\end{array}$ & 1935.230 & 1 & 1935.230 & 2.074 & & 0.155 \\
\hline & IL6 (pg/ml) & 292.344 & 1 & 292.344 & 4.137 & & 0.047 \\
\hline & $\begin{array}{l}\text { IL10 (pg/ml/ } \\
\text { day) }\end{array}$ & 21.000 & 1 & 21.000 & 0.316 & & 0.576 \\
\hline & $\mathrm{TNF} \alpha(\mathrm{pg} / \mathrm{ml})$ & 8.112 & 1 & 8.112 & 0.027 & & 0.869 \\
\hline & $\mathrm{IFN} \gamma(\mathrm{pg} / \mathrm{ml})$ & 588.747 & 1 & 588.747 & 5.570 & & 0.022 \\
\hline
\end{tabular}

Table 4 Comparison of mean vector components of biomarkers in case and control groups in ending study

\begin{tabular}{llllll}
\hline Effect & Value & $F$ & df & Error df & $p$ value \\
\hline Underlying diseases & & & & & \\
Pillai's trace & 0.260 & 2.310 & 7 & 46 & 0.042 \\
Wilks' lambda & 0.740 & 2.310 & 7 & 46 & 0.042 \\
Hotelling's trace & 0.352 & 2.310 & 7 & 46 & 0.042 \\
Roy's largest root & 0.352 & 2.310 & 7 & 46 & 0.042 \\
Group & & & & & \\
Pillai's trace & 0.349 & 3.526 & 7 & 46 & 0.004 \\
Wilks' lambda & 0.651 & 3.526 & 7 & 46 & 0.004 \\
Hotelling's trace & 0.537 & 3.526 & 7 & 46 & 0.004 \\
Roy's largest root & 0.537 & 3.526 & 7 & 46 & 0.004 \\
\hline
\end{tabular}

\section{Discussion}

To date, several clinical trials were in agreement that early transfusion of CP into severe COVID-19 patients after symptoms onset led to a reduction in mortality rate compared with other routine therapies. [24-30] However, some of them declared that its effect on the mortality rate was not statistically remarkable. [24, 27] In contrary to the most other clinical trials of CP therapy in past epidemics, Li et al.'s randomized trial showed that CP therapy (plus standard treatment), compared with merely standard treatment, did not significantly improve the mortality and/or the time of clinical recovery within 28 days. The time period of clinical improvement within 28 days ( 2.15 days) was shorter in the CP-treated group than the control group, but it was not statistically significant. In addition, the improvement of clinical symptoms occurred in 27 (51.9\%) cases in the CP-treated group vs. 22 (43.1\%) cases in the control group, which this difference was not statistically significant. [24] Based on Gharbharan et al.'s study results, no statistically significant differences were observed in improving the disease severity within 15 days and the mortality rate between the two groups. [27]

In this clinical and randomized controlled trial, we investigated the primary and secondary outcomes within a 60-day follow-up. Our results indicated that the mortality rate was lesser in CP-treated patients than the control group, but not significantly so. There is no statistically significant association between CP and the length of in-hospital stay; these results were consistent with the results of $\mathrm{Li}$ et al.'s and Gharbharan et al.'s studies. Moreover, despite the higher 
Table 5 Comparison of mean components of biomarkers in case and control groups with MANOVA analysis in ending study

\begin{tabular}{llcrrrr}
\hline Source & Dependent variable & Type III sum of squares & df & Mean square & $F$ & $p$ value \\
\hline Underly- & Absolute lymphocyte discharge & 1164216.382 & 1 & 1164216.382 & 1.04 & 0.313 \\
ing & CRP discharge & 379.451 & 1 & 379.451 & 0.89 & 0.349 \\
diseases & ESR discharge & 4457.221 & 1 & 4457.221 & 4.57 & 0.037 \\
& IL6 discharge & 369.125 & 1 & 369.125 & 8.60 & 0.005 \\
& IL10 discharge & 90.827 & 1 & 90.827 & 0.825 & 0.368 \\
& TNF $\alpha$ discharge & 786.569 & 1 & 786.569 & 2.651 & 0.110 \\
& IFN $\gamma$ discharge & 320.748 & 1 & 320.748 & 2.77 & 0.102 \\
Group & Absolute lymphocyte discharge & 2311289.317 & 1 & 2311289.317 & 2.06 & 0.157 \\
& CRP discharge & 66.901 & 1 & 66.901 & 0.157 & 0.693 \\
& ESR discharge & 26.685 & 1 & 26.685 & 0.027 & 0.869 \\
& IL6 discharge & 276.611 & 1 & 276.611 & 6.450 & 0.014 \\
& IL10 discharge & 207.643 & 1 & 207.643 & 1.886 & 0.176 \\
& TNF $\alpha$ discharge & 2715.029 & 1 & 2715.029 & 9.149 & 0.004 \\
& IFN $\gamma$ discharge & 523.316 & 1 & 523.316 & 4.530 & 0.038 \\
\hline
\end{tabular}

frequency of underlying diseases in the intervention group, the mortality rate in the CP-treated group was less. Hence, CP therapy may be more beneficial than standard treatments in general health improvement of severe patients.

In contrast to Li et al.'s and Gharbharan et al.'s studies, CP therapy plus standard drugs could significantly improve the 8-point WHO severity score (in the intervention group, mean score severity reduced 0.56 vs. 0.23 in the control group) (decrease of $36.7 \%$ in the intervention group against $26.7 \%$ in the control group below the score of 5) (Table 1). Considering the occurrence of some adverse events after CP transfusion in Li et al.'s clinical trial, despite the high quality of their plasma, their results may be affected by the low quality of transfusion and monitoring as confounders.

Published data accumulating from randomized controlled trials carried out around the world are different with respect to the target population, disease severity, outcome measures, and characterization of antibody status in donors and recipients. Most trials have focused on moderate to severely ill hospitalized patients, despite consistent findings and theoretical considerations that support early $\mathrm{CP}$ use relative to symptom onset. The large trial PLACID did not find a significant difference in outcome in moderately ill patients with COVID-19. However, a high proportion of recipients had low or absent antibodies. [34] A randomized, doubleblinded, placebo-controlled Argentinian multicenter trial enrolled hospitalized patients with COVID-19 pneumonia a median of 8 days from symptom onset and failed to show a clinical or mortality benefit for $\mathrm{CP}$, despite the use of high titer units. The Argentine study focused on COVID19 patients with pneumonia, so it is important to note that the same outcome cannot be extrapolated to milder cases of COVID-19. [35].

Furthermore, in the present clinical trial, the immunomodulatory effect of CP therapy on cytokine storm indices was evaluated, and the results were noteworthy. The CP therapy (plus standard drugs), compared to merely standard treatments, significantly increased the mean level of absolute lymphocytes and decreased the mean levels of IL- 6 , TNF- $\alpha$, and IFN- $\gamma$. In addition, the mean level of IL-10 was significantly increased after CP therapy on the day of discharge compared with its base level. Based on Duan et al.'s reports, the mean levels of CRP were significantly reduced on day four after $\mathrm{CP}$ transfusion, and the mean level of lymphocytes was increased. [26] By contrast, the base level of absolute lymphocyte did not change by day seven in Hegerova et al.'s study, [29] while the mean baseline CRP significantly decreased on day seven after CP therapy. In this regard, our findings showed that the mean levels of CRP and ESR were reduced in both groups, although there was no statistically significant difference between the two groups.

The main components of convalescent plasma include specific immunoglobulins against pathogens, albumin, complement, coagulation factors, and anti-inflammatory cytokines. [32] This convalescent plasma can provide immunomodulatory effects through the infusion of neutralizing antibodies (IgG and IgM-SARS-Cov-2) and anti-inflammatory cytokines into recipients' blood flow that lead to antiviral effect and neutralization of inflammatory cytokines, autoantibodies, and complement. [33] IL-4 and IL-10 are anti-inflammatory cytokines released following Th2 activation while TNF- $\alpha$, IL- $1 \beta$, and IL- 6 are pro-inflammatory cytokines secreted after Th1 type activation. [36] Previously, hung et al. reported that CP therapy in patients with influenza A (H1N1) led to a significant reduction of TNF $\alpha$, IL-6, and an increase of IL-10. [37, 38] In this regard, the findings of our study are confirmed this anti-inflammatory effect of convalescent plasma in severe COVID-19 patients too. Although standard treatments could also partially ameliorate the cytokine storm 
indices, the immunomodulatory effect of convalescent plasma was remarkably stronger and faster. Nevertheless, convalescent plasma could not strongly affect the mortality rate in spite of its significant ameliorative effect on cytokine storm, implies that the cytokine storm is not the sole major death factor and other factors may play a more important role in SARS-CoV-2 pathogenesis. In this regard, the presence of a systemic coagulation disorder in the lungs was reported [39], suggesting that the cause of death may not be the hyper-inflammatory state itself but instead a coagulopathy. The elevated levels of D-dimer and fibrinogen degradation products (FDP) in COVID-19 patients are indicating of coagulopathy, a disorder that has been shown to be associated with a high risk of mortality [40]. Of course, the possible role of external risk factors on the $\mathrm{CP}$ treatment outcomes should also be considered in the clinical trials, e.g., patients' stress, the physician's clinical skill level, the quality of monitoring, and the plasma transfusion speed.

\section{Study strengths and limitations}

The limitation of this study was the impossibility of increasing the sample size due to a decrease in the number of eligible volunteer patients during the study period. The strengths of this study were randomization and single blinding to minimize the impact of possible confounders on the research results.

\section{Conclusion}

The convalescent plasma has a remarkable immunomodulatory and antiviral potential to improve cytokine storm and 8-point WHO severity score in COVID-19 patients.

Supplementary Information The online version contains supplementary material available at https://doi.org/10.1007/s11739-021-02734-8.

Acknowledgements We would like to gratefully acknowledge the research deputy of Ahvaz Jundishapur University of Medical Sciences who supported this work. We also appreciate Dr. Abolnezhadian, Ms. Leila Kord Zangeneh, Mr. Nader M.Ch, and the personnel of the Blood Transfusion Organization for their helping.

Author contributions MP conceptualization, supervision, investigation, and funding acquisition; MS supervision, project administration, methodology, and writing - review and editing; PE and HA methodology and resources; AGB and BS investigation; PMC investigation, data curation, writing — original draft, and writing — review and editing; AF resources and coordination of plasma donation plan; MGB and AF resources; MAJF, GAK, and MA laboratory consultation; ER, MT and FY clinical consultation; RH coordination for blood plasma separation device.

\section{Declarations}

Ethical approval All the procedures performed in studies involving human participants were in accordance with the ethical standards of the national research committee and with the 2008 Helsinki Declaration and its later amendments or comparable ethical standards. This hospital-based, parallel-group and randomized controlled trial was approved by the Ethics Committee of Ahvaz Jundishapur University of Medical Sciences, Ahvaz, Iran with Ethical Code: IR.AJUMS.REC.1399.003 and IRCT20200310046736N1.

\section{References}

1. Huang C, Wang Y, Li X et al (2020) Clinical features of patients infected with 2019 novel coronavirus in Wuhan China. Lancet 395:497-506

2. Han H, Ma Q, Li C et al (2020) Profiling serum cytokines in COVID-19 patients reveals IL-6 and IL-10 are disease severity predictors. Emerg Microbes Infect 9(1):1123-1130

3. Chen L, Liu HG, Liu W et al (2020) Analysis of clinical features of 29 patients with 2019 novel coronavirus pneumonia. Zhonghua Jie He He Hu Xi Za Zhi 43:E005

4. Xu Z, Shi L, Wang Y et al (2020) Pathological findings of COVID-19 associated with acute respiratory distress syndrome. Lancet Respir Med. https://doi.org/10.1016/S2213-2600(20) 30076-X

5. Channappanavar R, Perlman S (2017) Pathogenic human coronavirus infections: causes and consequences of cytokine storm and immunopathology. Semin Immunopathol 39:529-539

6. Wang Z, Yang B, Li Q et al (2020) Clinical features of 69 cases with coronavirus disease 2019 in Wuhan, China. Clin Infect Dis 71(15):769-777

7. Chen G, Wu D, Guo W et al (2020) Clinical and immunological features of severe and moderate coronavirus disease 2019. J Clin Invest 130:2620-2629

8. Michot JM, Albiges L, Chaput N et al (2020) Tocilizumab, an anti-IL6 receptor antibody, to treat Covid-19-related respiratory failure: a case report. Ann Oncol 31:961-964

9. Zhang X, Song K, Tong F et al (2020) First case of COVID-19 in a patient with multiple myeloma successfully treated with tocilizumab. Blood Adv 4:1307-1310

10. Odievre MH, de Marcellus C, Le Ducou Pointe H et al (2020) Dramatic improvement after tocilizumab of a severe COVID-19 in a child with sickle cell disease and acute chest syndrome. Am J Hematol. https://doi.org/10.1002/ajh.25855

11. Chen N, Zhou M, Dong X et al (2020) Epidemiological and clinical characteristics of 99 cases of 2019 novel coronavirus pneumonia in Wuhan, China: a descriptive study. Lancet 395:507-513

12. Wang D, Hu B, Hu C et al (2020) Clinical characteristics of 138 hospitalized patients with 2019 novel coronavirus-infected pneumonia in Wuhan China. JAMA 323:1061-1069

13. Lenzer J (2020) Covid-19: US gives emergency approval to hydroxychloroquine despite lack of evidence. BMJ 369:m1335

14. World Health Organization. Coronavirus Disease 2019(COVID19) Situation Report-41: Data as Reported by 10AM CET 01 March 2020. Available online at: https://www.who.int/docs/ default-source/coronaviruse/situation-reports/20200301-sitrep41-covid-19.pdf?sfvrsn=6768306d_2.

15. Tang Y, Liu J, Zhang D et al (2020) Cytokine storm in COVID19: the current evidence and treatment strategies. Front Immunol 11:1708

16. Rieder M, Wengenmayer T, Staudacher D et al (2020) Cytokine adsorption in patients with severe COVID-19 pneumonia 
requiring extracorporeal membrane oxygenation. Crit Care 24:435. https://doi.org/10.1186/s13054-020-03130-y

17. Tan W, Li S, Yiyang C et al (2020) Clinical efficacy analysis of 50 cases of corona virus disease 2019 in traditional Chinese medicine. Jilin J Chin Med 40:281-285

18. Kai-tao Y, Ming-yu L, Xin L et al (2020) Retrospective clinical analysis on treatment of novel coronavirus-infected pneumonia with traditional Chinese medicine Lianhua Qingwen. Chin J Exp Tradition Med Formul 26:8-12

19. Hou L, Huang H (2016) Immune suppressive properties of artemisinin family drugs. Pharmacol Therap 166:123-127

20. Mair-Jenkins J, Saavedra-Campos M, Baillie JK et al (2015) The effectiveness of convalescent plasma and hyperimmune immunoglobulin for the treatment of severe acute respiratory infections of viral etiology: a systematic review and exploratory meta-analysis. J Infect Dis 211:80-90

21. Planitzer CB, Modrof J, Kreil TR (2007) West Nile virus neutralization by US plasma-derived immunoglobulin products. J Infect Dis 196:435-440

22. Rojas M, Monsalve DM, Pacheco Y et al (2020) Ebola virus disease: An emerging and re-emerging viral threat. J Autoimmun 106: 102375

23. Garraud O, Heshmati F, Pozzetto B et al (2016) Plasma therapy against infectious pathogens, as of yesterday, today and tomorrow. Transfus Clin Biol 23:39-44

24. Li L, Zhang W, Hu Y et al (2020) Effect of convalescent plasma therapy on time to clinical improvement in patients with severe and life-threatening COVID-19: a randomized clinical trial. JAMA 324(5):460-470

25. Abolghasemi H, Eshghi P, Cheraghali AM et al (2020) Clinical efficacy of convalescent plasma for treatment of COVID-19 infections: results of a multicenter clinical study. Transfus Apher Sci. 59(5): 102875

26. Duan K, Liu B, Li C et al (2020) Effectiveness of convalescent plasma therapy in severe COVID-19 patients. ProcNatl Acad Sci. 117(17):9490-9496

27. Gharbharan A, Jordans CCE, GeurtsvanKessel C et al (2020) Convalescent plasma for COVID-19. A randomized clinical trial. MedRxiv. https://doi.org/10.1101/2020.07.01.20139857

28. Salazar E, Perez KK, Ashraf M et al (2020) Treatment of COVID-19 patients with convalescent plasma. Am J Pathol 190(8):1680-1690

29. Hegerova L, Gooley T, Sweerus KA, Maree CL, Bailey N, Bailey $M$ et al (2020) (2020) Use of convalescent plasma in hospitalized patients with covid-19—case series. Blood 136(6):759-762
30. Xia X, Li K, Wu L et al (2020) Improved clinical symptoms and mortality on severe/critical COVID-19 patients utilizing convalescent plasma transfusion. Blood 136(6):755-759

31. Piechotta V, Chai KL, Valk SJ, Doree C, Monsef I, Wood EM et al (2020) Convalescent plasma or hyperimmune immunoglobulin for people with COVID-19: a living systematic review. Cochrane Database Syst Rev. 7(7):013600

32. Benjamin RJ, McLaughlin LS (2012) Plasma components: properties, differences, and uses. Transfusion 52(Suppl. 1):9S-19S

33. Lunemann JD, Nimmerjahn F, Dalakas MC (2015) Intravenous immunoglobulin in neurology-mode of action and clinical efficacy. Nat Rev Neurol 11:80-89

34. Agarwal A, Mukherjee A, Kumar G, Chatterjee P, Bhatnagar T, Malhotra P (2020) Convalescent plasma in the management of moderate covid-19 in adults in India: open label phase II multicentre randomised controlled trial (PLACID Trial). BMJ. https:// doi.org/10.1136/bmj.m3939

35. Simonovich VA, Burgos Pratx LD, Scibona P, Beruto MV, Vallone MG, Vázquez C et al (2020) A randomized trial of convalescent plasma in Covid-19 severe pneumonia. N Engl J Med. 24:NEJMoa2031304

36. Choghakabodi PM, Pouladzadeh M, Haybar H et al (2020) Biological whistleblowers for silent myocardial ischemia: diagnostic and prognostic approach. Recenti Prog Med 111(7):415-425

37. Hung IFN, Toe KKW, Lee C-K et al (2013) Hyperimmune IV immunoglobulin treatment: a multicenter double-blind randomized controlled trial for patients with severe 2009 influenza A (H1N1) infection. Chest 144(2):464-473

38. Hung IF, To KK, Lee C-K et al (2011) Convalescent plasma treatment reduced mortality in patients with severe pandemic influenza A (H1N1) 2009 virus infection. Clin Infect Dis 52(4):447-456

39. Zhang Y, Xiao M, Zhang S, Xia P, Cao W, Jiang W et al (2020) Coagulopathy and antiphospholipid antibodies in patients with Covid-19. N Engl J Med 382(17):e38

40. Magro G (2020) Cytokine storm: is it the only major death factor in COVID-19 patients? Coagul role Med Hypotheses 142:109829

Publisher's Note Springer Nature remains neutral with regard to jurisdictional claims in published maps and institutional affiliations.

\section{Authors and Affiliations}

\section{Mandana Pouladzadeh ${ }^{1}$ Mehdi Safdarian ${ }^{2}$ (1) - Peyman Eshghi ${ }^{3,4}$. Hassan Abolghasemi ${ }^{5}$. Alireza Ghorbani bavani ${ }^{6}$. Behnam Sheibani $^{7}$. Parastoo Moradi Choghakabodi ${ }^{8}$ (D) Abdolaziz Feghhi ${ }^{9} \cdot$ Mehri Ghafourian Boroujerdnia $^{10}$. Arash Forouzan ${ }^{11}$. Mohammad Ali Jalali Far ${ }^{8}$. Gholam Abbas Kaydani ${ }^{12}$. Elham Rajaei ${ }^{13}$. Mansour Amin ${ }^{14,15}$. Mehdi Torabizadeh ${ }^{16} \cdot$ Farid Yousefi $^{15} \cdot$ Reza Hadaddezfuli ${ }^{17}$}

1 Emergency Medicine Department, School of Medicine, Ahvaz Jundishapur University of Medical Sciences, Ahvaz, Iran

2 Nanotechnology Research Center, Ahvaz Jundishapur University of Medical Sciences, Ahvaz, Iran

3 Pediatric Congenital Hematologic Disorders Research Center, Shahid Beheshti University of Medical Sciences, Tehran, Iran
4 Blood Transfusion Research Center, High Institute for Research and Education in Transfusion Medicine, Tehran, Iran

5 Applied Microbiology Research Center, Systems Biology and Poisonings Institute, Baqiyatallah University of Medical Sciences, Tehran, Iran

6 Radiology Department, Abadan University of Medical Sciences, Abadan, Iran 
7 Infectious Diseases Department, Ahvaz Jundishapur University of Medical Sciences, Ahvaz, Iran

8 Thalassemia and Hemoglobinopathy Research Center, Health Research Institute, Ahvaz Jundishapur University of Medical Sciences, Ahvaz, Iran

9 Blood Transfusion Organization, Ahvaz, Khuzestan, Iran

10 Department of Immunology, School of Medicine, Ahvaz Jundishapur University of Medical Sciences, Ahvaz, Iran

11 Department of Emergency Medicine, Imam Khomeini General Hospital, Ahvaz Jundishapur University of Medical Sciences, Ahvaz, Iran

12 Department of Laboratory Sciences, Allied Health Sciences School, Ahvaz Jundishapur University of Medical Sciences, Ahvaz, Iran
13 Department of Internal Medicine, Ahvaz Jundishapur University of Medical Sciences, Ahvaz, Iran

14 Department of Microbiology, School of Medicine, Ahvaz Jundishapur University of Medical Sciences, Ahvaz, Iran

15 Infectious and Tropical Diseases Research Center, Health Research Institute, Ahvaz, Iran

16 Golestan Hospital Clinical Research Development Unit, Ahvaz Jundishapur University of Medical Sciences, Ahvaz, Iran

17 Ahvaz Jundishapur University of Medical Sciences, Ahvaz, Iran 